\title{
Mechanism of Perceptual Categorization in the Pre-Linguistic Period
}

Tamami SUDO $^{\dagger, \dagger \dagger}$, Student Member and Ken MOGI ${ }^{\dagger \dagger}$, Nonmember

\begin{abstract}
SUMMARY In this study, we conducted a series of experiments using stimuli characterized by various attributes in order to understand the categorization process in an infant's pre-linguistic development. The infants are able to assign the same label to members within the same category by focusing attention on specific features or functions common to the members. The ability to categorize is likely to play an essential role in an infant's overall cognitive development. Specifically, we investigated how the infants use different strategies in the process of linguistic categorization. In one strategy, members of a single category are derived from perceptual similarities within the most representative members, i.e., the prototypical members. Alternatively, each membership is established by referring to the linguistic labels for each category provided by the caretaker, in a symbol grounding process. We found that the infant is able to employ these strategies in a flexible manner in its development. We discuss the interplay between different cognitive strategies, including the prototype effects in the infant's cognitive development and the implications for cortical mechanism involved.

key words: categorization, word-learning, prototype effect, preferential looking
\end{abstract}

\section{Introduction}

It is known that infants are aware of the category of objects in daily life before explicit vocabulary acquisition starts. Even though the various attributes such as color, texture, size are not the same for individual objects, infants are able to perceive them as belonging to a single category. The infants are able to assign the same label to members within the same category by focusing attention on specific features or functions common to the members. It has been suggested that the ability of categorization is likely to play an essential role in its overall cognitive development.

In this study, we conducted a series of experiments using stimuli which have various attributes in order to understand the process of such a categorization in an infant's prelinguistic development. We found that the infant is able to employ several strategies in a flexible manner in its development. We consider two possible cognitive processes of linguistic categorization. One possible scenario is that certain members of the categories are derived from similarities with the most representative members of a category (prototypical members) [1]. Alternatively, the coupling of each category member-ship with relevant labels can be traced to

Manúscript received September 7, 2007.

Manuscript revised December 14, 2007.

${ }^{\dagger}$ The author is with Interdisciplinary Graduate School of Science and Engineering, Tokyo Institute of Technology, Yokohamashi, 226-8502 Japan.

${ }^{\dagger \dagger}$ The authors are with Sony Computer Science Laboratories, Inc., Tokyo, 141-0022 Japan.

DOI: 10.1093/ietisy/e91-d.6.1649 the symbol grounding problem [2]. Our results are consistent with an interpretation that the cortical system involved in language development is inseparably linked with prototype effects in categorization.

\section{Overview of Word-Learning}

The human linguistic system is complex and seems to be arbitrary in the choice of specific labels to the objects and concepts. Nevertheless, young children become fluent speaker of the native language in a short period. Acquiring this complex linguistic system in a fairly short time is an absolute marvel, reflected in the characteristic human cognitive development [3]. It has been known that young children are able to master the use of the native language without any special training. An environment where they can participate in the usual social exchange is sufficient. During the development in the period from 1.5 to 6 years after birth, infants add new words to their vocabulary at an average rate of 8 words a day. In this period, the infants are not provided with a definition of the meaning or the usage of the new words. Infants learn by inferences from the conversations that they encounter.

Based on empirical evidences, it is unacceptable to explain the learning of language in a way that is dependent on a solid and formal acquisition of each word. If the acquisition of the name of an object is done in a one by one examination of the unique properties of the objects in relation to all the other objects in the world, vocabulary acquisition should take an enormous time. It is logically impossible to make reasonable inferences from only a limited amount of information on the objects that a particular word is apparently referring to. Despite these seeming theoretical impossibilities, children can acquire a particular meaning from the enormous possible set of meanings of the words referring to the objects [4].

At least one thing is clear in the children's word learning. When children listen to a new word, they never explore the whole domain of the logically possible meaning of the particular word in question. In the majority of cases children "guess" the meaning of the words which they listen to for the first time and assign a temporary meaning to that word after making proper reasoning. Such a temporary assignment of the meaning of the word has been called "fast mapping" [5].

As a possibly effective method of dissolving the apparent difficulty in coming to an understanding of an infant's language acquisition, and accounting for the seemingly 
effortless way in which children conduct fast mapping, recently the concept of cognitive constraint has entered the mainstream approach.

The cognitive constraint here refers to the conceptual scheme that children retain internally when they encounter a new word. The cognitive constraint effectively narrows the range of search that needs to be done in the acquisition of new words [6], [7]. Categorization can be characterized as a cognitive process in which the domain of its reference is defined. The cognitive constraint becomes an important element in the process of categorization. The concept of cognitive constraint is introduced in a marked contrast with the philosophy of behavioral psychology in which children's learning and development are attained through the inputs and feedbacks from the outside. The cognitive constraints are metaphors with which the cognitive linguists try to account for an infant's inherent ability of language development in the pre-linguistic period.

\section{Category Formation in Infants}

It is known that infants are aware of the category of objects in daily life before explicit vocabulary acquisition starts. Infants only a few months old are able to separate complex visual stimuli into generic object categories [8]. For instance, infants are able to recognize the various "cars" - pictures of cars in books, real cars running on the street etc., as belonging to a single category. Even though the various attributes such as color, texture, size are not the same for a real car and a picture car, they are able to perceive them as belonging to a single category. Several studies have shown that infants from at least 3 months old can discriminate between basic object categories [9]. In such experiments, called habituation task, an infant is shown a stimulus until they are bored of it, and look at it no longer. Then this habituated stimulus is presented alongside a test stimulus. If the child now looks at the novel stimulus more than the habituated one, we can conclude that they can recognize the difference between them.

In addition, Nazzi and Gopnik reported infants' ability to form new object categories based on either visual or naming information alone [10]. Infants as young as 20 months can use naming cues to form completely new object categories in the absence of any other cues, even when the objects are completely dissimilar to each other.

As a result of this categorization process, children as young as 30 months old can possess the ability to classify objects markedly different in terms of specific sensory properties as belonging to a single category [11], [12]. It has been suggested that the ability of categorization is a metaknowledge tightly coupled with an infant's language acquisition, and is likely to play an essential role in its overall cognitive development.

Infants are very hard to conduct psychological experiments on. They get bored, they can not understand instructions, they cry, and they can't press buttons. However, one of the few relatively sophisticated abilities that babies have are their visual abilities. A baby will pay attention and look at anything that is new and interesting. This is the root of the preferential looking tasks, as pioneered by Fantz [13], [14]. Data based on preferential looking have shown that infants are able to differentiate between a striped black and white pattern and one which is uniformly grey and of the same total luminance. They spent a greater proportion of their time looking at the stripes.

Infants often understand many more words than they can say, but may be unable to follow instructions or understand what is required of them. The preferential looking paradigm is one of the relatively tractable techniques that allow us to investigate the cognitive ability of very young children, even those who are not yet able to speak or understand complex instructions. The paradigm essentially requires the child to listen to sentences and watch screens. This particular approach is based on the fact that infants tend to look at scenes corresponding to sentences that they hear. In the present study, therefore, we also employed this methodology for phonological association task as we will describe below.

\section{Phonological Association Task}

Word-object association is the process in which objects that exist in the environment is associated with the words. This cognitive process is an important ability acquired in the prelinguistic period, is necessary for facilitating word learning, and is involved in the process of categorization.

When infants in the pre-linguistic period encounter various situations where the usage of language is involved, how do they make the word-object association?

For example, a familiar object such as an apple can take various forms, when it is cut and dressed. The child is apparently able to recognize these physically different entities as belonging to the same category. In order to clarify this point, we conducted a series of experiments.

\subsection{Method}

\subsubsection{Participants}

Twenty 14 to 24 month-old Japanese infants $(\mathrm{M}=21 \mathrm{~m} 0 \mathrm{~d})$ participated in the study. There were eleven boys and nine girls. The infants sat facing the screen, placed on their mother's lap.

\subsubsection{Stimuli}

As typical examples of objects in the environment, foods, creatures, and artifacts are considered, represented by "apple", "fish", and "car". Three colored stimuli (pictures or photos) are used for each category (i.e., 9 stimuli in total). Pictures of target objects were varied in the salient features such as color, form, and size. For example, the apple was given either as a whole, cut half, or dressed as a rabbit (given in Fig. 1 and Table 1). 

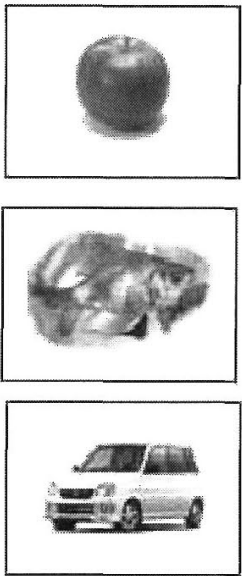

pie.1
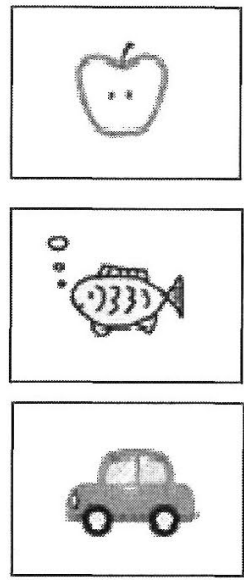

pie.2
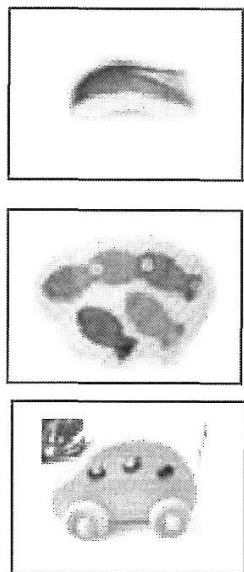

pie.3
Fig.1 Stimulus objects.

Table 1 Whole list of visual stimuli.

\begin{tabular}{ll}
\hline Category-apple & $\begin{array}{l}\text { Whole apple photo } \\
\text { Half apple picture } \\
\text { Rabbit-shaped apple photo }\end{array}$ \\
\hline \multirow{2}{*}{ Category-fish } & $\begin{array}{l}\text { Line drawing fish } \\
\text { Goldfish photo } \\
\text { Cloth fish photo }\end{array}$ \\
\hline & $\begin{array}{l}\text { White car photo } \\
\text { Red car picture } \\
\text { Woody toy car photo }\end{array}$ \\
\hline
\end{tabular}

The variations in visual properties were designed to fall within range of those encountered in daily life, rather than to follow physically specified criteria for variation.

Each of the categories (apple, fish, car) consists of three Japanese moras. Phonological word familiarity and number of moras were matched based on the database of Japanese words [15].

A pair of pictures picked up from each category was projected on the screen from a laptop.

The infants were seated approximately $360 \mathrm{~cm}$ away from the screen that were $39 \mathrm{deg}$ of visual angle wide and $24 \mathrm{deg}$ high, and the paired pictures were presented on the screen at certain visual angles shown in Fig. 2.

\subsubsection{Procedure}

First, a white screen was presented for 1 second. Continuously, each pair of pictures was presented for 8 seconds. After that another white screen was shown for 1 second. The stimulus design is given in Fig. 3. 9 trials were conducted. As the two pictures in different categories were shown on the screen, the experimenter seated next to subjects asked (in Japanese) " 'Ringo' [the name of one of the categories] wa dotti?" [Which is (an) apple?] virtually simultaneously.

The order of stimulus presentation was counterbalanced. The matching pattern with the phonological words

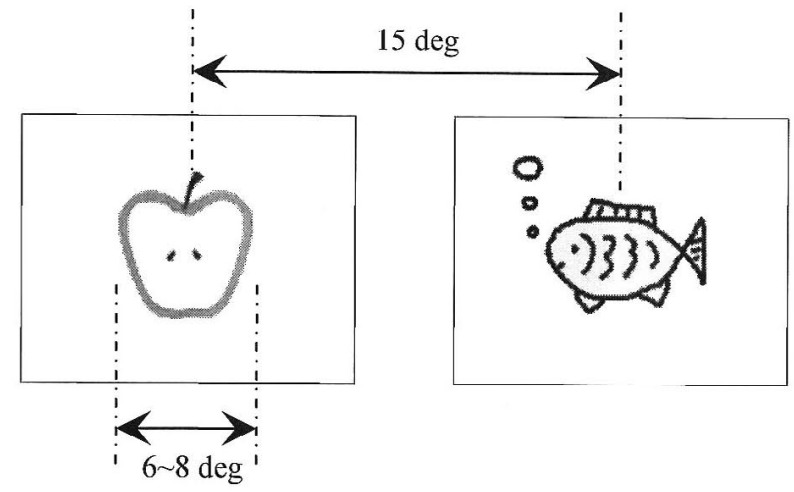

Fig. 2 Scale of visual stimuli.

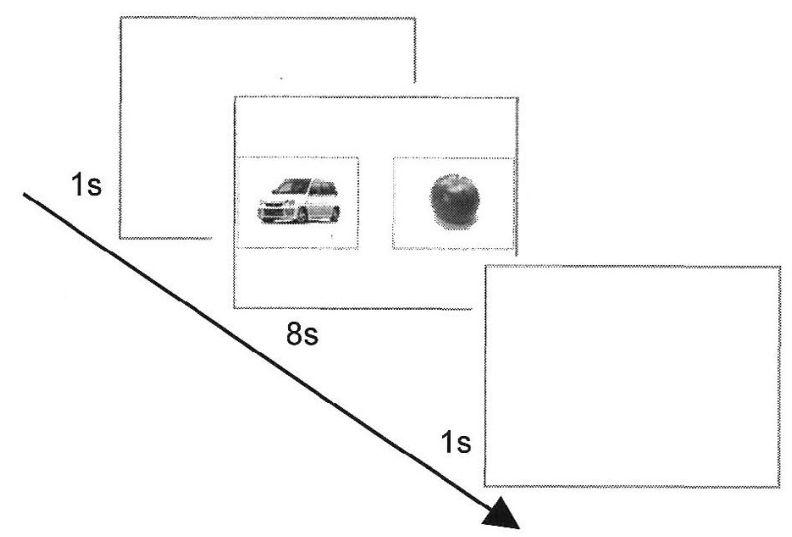

Fig. 3 Sample of presentation pattern.

(right match, left match, mismatch) were randomized.

During the experiment, accompanying mothers were instructed not to suggest a particular object by means of finger pointing, and to refrain from any action inducing the infants to pay attention in any particular way. When such a maternal behavior was observed, the data were excluded from the analysis.

As mentioned previously, in preferential looking experiments, an infant will be shown two objects projected onto a screen, and the experimenter will measure the amount of time a child spends looking at each object. As a result, one of two behaviors will be observed from this situation. Either the infant looks at one object for a significantly greater amount of time than it does the other, or both objects are looked at for approximately the same amount of time.

Images from the frontal and back views were captured by two video cameras. The two images were recorded within a single frame after being merged through a device. After the experiment, one of the experimenters played the video to analyze the results and determined the looking time for each object. Trials in which infants looked away from the screen more than $75 \%$ of the whole session were excluded from further analysis.

By measuring the mean looking time, we investigated the ability of the infants to generalize over image variations in associating the images with an audio stimulus. 


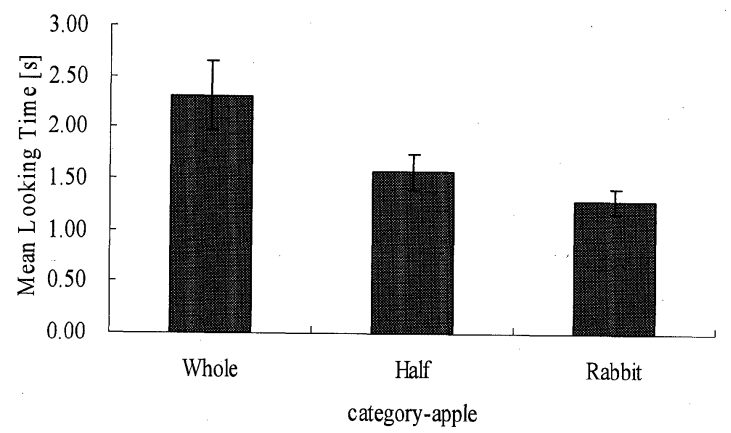

(a)

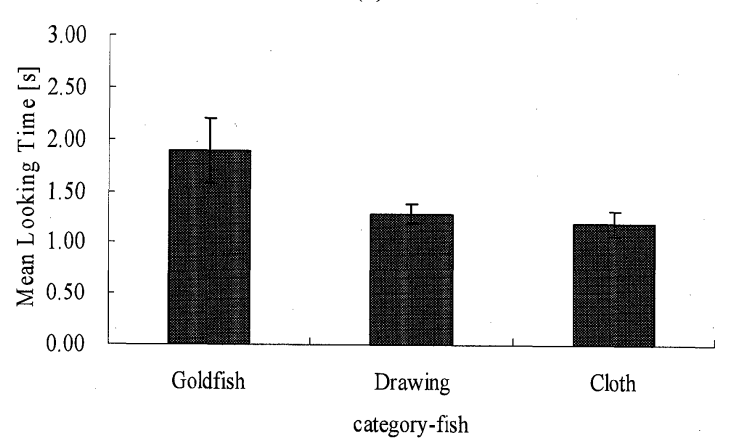

(b)

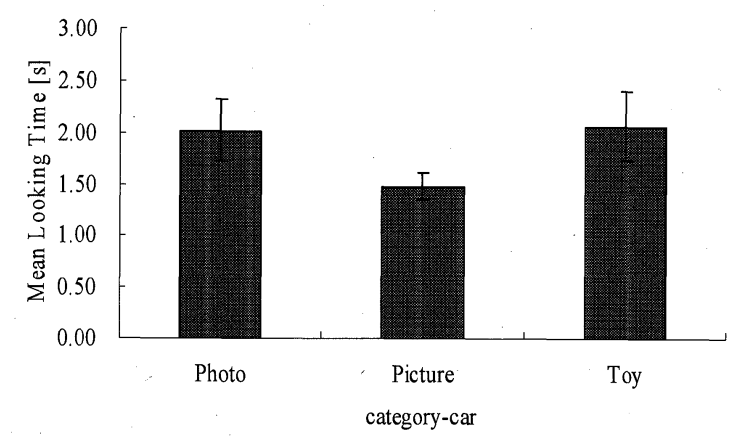

(c)

Fig. 4 Averaged mean looking time of target objects in all trial (a) apple, (b) fish, (c) car.

\subsection{Results}

We analyzed the infants' looking time at the stimuli presented on the screen. The mean looking times for all the objects presented were calculated, after excluding trials which did not satisfy criteria described in the method, resulting in different numbers of trials for each category.

Significant effects of the physical shape of the object were observed except for category-car (artifact) (apple: $F(2,31)=5.690, p=0.008$ (Fig. 4 (a)), fish: $F(2,30)=$ 4.116, $p=0.026$ (Fig. 4 (b)), car: $F(2,34)=1.561, p=0.225$ (n.s.) (Fig. 4 (c)), total: $F(2,105)=7.734, p<0.001)$.

A repeated measure ANOVA with pairing objects (target vs. other) revealed a significant difference (apple: $F(1,72)=67.21, p<0.001$, fish: $F(1,66)=64.46, p<0.001$, car: $F(1,72)=83.28 p<0.001$, total: $F(1,214)=205.9$, $p<0.001$ ) (Fig. 5). Here, "target" refers to the object in match with the phonological word, while the "other" refers

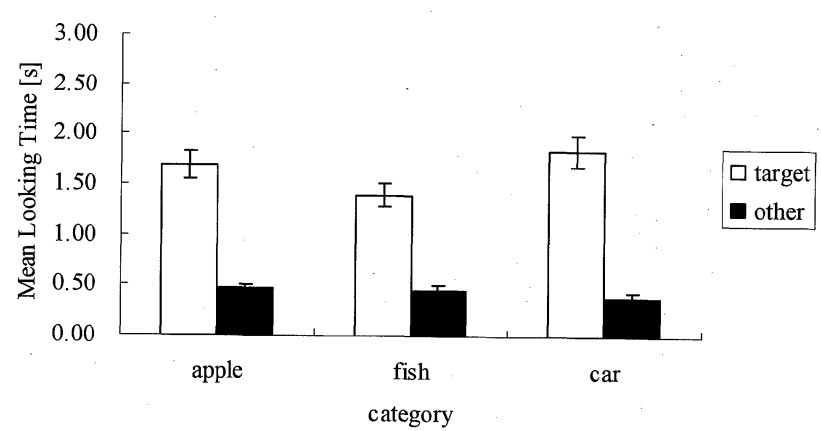

Fig. 5 Preference Scale (target vs. other). Averaged mean looking time of each categories in all trial.

to the alternative object. The result (Fig. 5) indicates that the infants are able to discriminate the "target" from the "other", suggesting they are able to categorize with the aid of phonological labels.

The experiment is based on a preferential looking paradigm, and does not address the explicit abilities for the infants to use the acquired category in a linguistic or prelinguistic context. With that limitation in mind, these results are consistent with the interpretation that the infants are apparently able to perceive physically different objects as belonging to a single category even before explicit verbal ability emerges.

The particular objects presented are taken from the categories of food, creature, and artifact. The ability to categorize might therefore be a general one, although no conclusions can be drawn at this stage about the generality of the results.

This experiment alone does not tell us how the infants are able to conduct this pre-linguistic categorization. There could be two major ways of realizing the categorization. Firstly, the infants could use the perceptual similarities between the various forms of apple. Even when the apple is cut in half or dresses as a rabbit, there are visual similarities (such as the color or the texture) that the infant could utilize in the categorization process.

Alternatively, the infants can use the single auditory label as common to the various appearances of each category. The first strategy is more perceptually based, whereas the second strategy is tightly coupled with the linguistic maturity to come. The infants could utilize these different strategies to a varied extent at different stages of development.

In order to clarify this point, we conducted a second series of experiments, where we presented two different age groups of infants with several pictures of different objects in a contingent context.

\section{Naming and Visual-Pairing Task}

In this experiment, we investigated whether there are significant changes in the ability for categorization and naming in formative stage. The key question was whether the infants depended on visual information or linguistic information when they perceived the different forms of an object 
(e.g. apple) as belonging to the same category.

In early' findings, Nazzi and Gopnik investigated infants' ability to use naming cues to form new object categories in the absence of any other cue at two different ages (16 and 20 months) [10]. They used the objects selected in such a way that the infants would be unfamiliar with them and would not perceive them with natural linguistic labels. They reported that infants at both ages showed evidence of using visual information to categorize the objects, while only the older ones used naming information. Thus they indicated that infants as young as 20 months can use the non-obvious cue of naming to categorize objects.

Therefore, we modified the experimental design of linguistic categorization trial by Nazzi et al., by using familiar and well-known objects.

\subsection{Method}

\subsubsection{Participants}

Twenty Japanese infants of two age groups participated in the study. They were fallen into two groups of the same number. Each group had an equal number of boys and girls. 10 of them were aged round 15 months $(M=15 \mathrm{m6d})$, and the remaining 10 were aged 21 months $(M=21 \mathrm{~m} 3 \mathrm{~d})$. Some additional infants of each age were tested, but failed to complete the session. Each of infants was seated on their mother's lap across a table from the experimenter.

\subsubsection{Stimuli}

Seven pairs of pictures were picked up from all of visual stimuli used in previous experiment and projected on the screen as same as in phonological association task.

\subsubsection{Procedure}

In the warm-up phase, the infants in the presence of mother looked at prototypical pictures or photos of "apple", "fish", and "car" posted on a sketchbook. These pictures were not identical to, but the same color or shape as those that induced the maximum mean looking time in the first experiment. This phase lasted for a few minutes. The warm-up phase was designed to ascertain that the stimuli presented were familiar and well-known for the subjects, and to make the familiarity as equal as possible among the subjects.

In the test phase, the two pictures in different categories were given on the screen as same as in previous experiment. Then the experimenter asked them a series of questions. In the naming task, the experimenter asked directly like (in Japanese) " 'Ringo' [object's name] wa dotti?" Which is (an) apple?]. In the visual-pairing task, the experimenter showed another visual stimulus in the sketchbook that was used as the prototypical picture of the target category in the warm-up phase and asked the question (in Japanese) "Kore to onaji mono wa dotti?" [Which is the same thing as this?] simultaneously. The infants answered by pointing to one of
Table 2 Mean numbers of correct responses.

\begin{tabular}{ccc}
\hline & Naming & Visual \\
\hline $\begin{array}{c}\text { Younger } \\
(15 \text { month })\end{array}$ & $5.63^{*}$ & 3.75 \\
\hline $\begin{array}{c}\text { Elder } \\
(21 \text { month })\end{array}$ & $6.43^{*}$ & $5.14 *$ \\
\hline & $* p<0.001$
\end{tabular}

the pictures on the screen. Both procedures were repeated for the seven sets of pictures in random order.

\subsection{Results}

The results of the naming task and the visual pairing task were analyzed separately. For each trial, the infants were given a score of 1 when they chose the correct object, and a score of 0 when they chose the other or none. Mean total scores per age and task are given in Table 2. Twotailed $t$-tests were conducted to determine whether infants were choosing the correct picture significantly above the chance level. In the naming task, significantly high correct rates above chance were revealed in both the younger group $(\mathrm{M}=5.63, t(9)=8.30, p<0.001)$ and the elder group $(\mathrm{M}=6.43, t(9)=11.6, p<0.001)$. In the visual-pairing task, these analyses revealed that infants of the younger group were responding at chance $(\mathrm{M}=3.75, t(9)=0.51$, $p=0.62$ ), while infants of the elder group were responding above chance level $(\mathrm{M}=5.14, t(9)=9.14, p<0.001)$.

The marked difference in the correct rate for the visual and naming tasks is interesting. These results are consistent with the interpretation that infants give priority to linguistic information over the visual information when the presented stimuli are well known to them.

Based on these results, we consider it likely that a fair level of success in the naming task is to be attributed to the familiarity of objects. This is caused by familiarity which is tightly coupled with internal representation. On the other hand, failure of the visual task in the younger infants may be due to the difficulty in forming a common category based on perceptual similarities in the absence of an auditory label.

\section{Discussion}

In the experiment of naming and visual-pairing task, both familiar and common object were presented for stimuli. For this reason, a sharp contrast was found between our experiment and Nazzi's experiment. The bottom line of this paradoxical aspect is that infants' categorization ability guided by linguistic information will be developed quite early in their developmental phase, even though Nazzi's experiment demonstrates that infants can use language to form object categories at least at 20 months after birth. In addition to the result of Nazzi and Gopnik, infants exhibit more robust abilities to categorize based on phonological labels in our study when they are given the objects that they are quite familiar with. 
In the present study, the language database was applied for stimulus evaluation. Such a parametric approach like quantitatively-measured word familiarity might facilitate understanding mechanism of category formation in the order of developmental phase.

Infants in the earlier age group in this study exhibited only a chance level performance in the visual pairing task. This result might be due to the inability of the infants to categorize unless an explicit label was given by means of, e.g., phonological labels. Moreover, the failure might be due to the lack of generalization in terms of sensory properties in the process of categorization. The gradual development of categorization in infants would proceed in such a manner as to satisfy the constraints suggested by the present study.

Within the fields of cognitive psychology and cognitive linguistics, categorization has become a major field of study and the prototype effect is focused as the characteristic features of categorization [16]. Prototype theory is a model of graded categorization in cognitive science, where some members of a category are more central than others. This theory also plays a central role in language acquisition, as part of the mapping from phonological structure to semantics. Prototype effect would consider a category like bird as consisting of different elements which have unequal status - e.g. a robin is more prototypical of a bird than, say a penguin. This leads to a graded notion of categories, which is a central notion in many models of cognitive science and cognitive semantics [1].

The results from the experiment of phonological association task showed significant effects of the physical shape of the object. In the present study, the phonological association task was conducted to study the association between linguistic labels presented as auditory stimuli and the corresponding objects presented as visual stimuli. This association may be mediated through an internal prototype representation of the object's category. From the aspect of prototype effects, we may conclude that the relatively more representative members in the category are easier to invoke the prototypical member in the inner representation. Thus, infants were able to find appropriate objects out of the stimuli presented and to associate them with the auditory labels. The different behavior observed in the category of "car" (an artifact) might be due to the familiarity with common categories represented by, for example, toys for children.

The infants' behavior could have been affected by perceptual similarities between the presented stimuli within a single category. The detailed nature of such an effect can be clarified by introducing parameters of control of quantitative similarity relations between the stimuli. We plan to conduct such a scheme in future studies.

It would be possible to clarify the nature of perceptual similarities between the objects and the strength of links with labels for categories through studies based on the prototypical effects. It would be then interesting to formulate a model of the development of categorization in infants based on the prototypical effect. We plan to explore possibilities for models of cognitive development based these considerations.

In addition, we may consider a kind of symbol grounding problem. In the visual-pairing condition, test stimuli given with the question were treated as one of the most typical member of the target categories that evoked the prototypical member. It is suggested that their similarity enabled to analogize other members from these stimuli.

Finally, the linguistic labels given in the naming task made it easier to recall the prototype within each category than the similar members in the visual-pairing task. The different strategies used in categorization are expected to vary according to children's stage of development.

It has been much debated whether children weigh shape similarity more heavily than conceptual similarity, invisible to the eye, such as taxonomic relatedness or functional commonality [17]. Through several developmental phases, conceptual similarity seems to take precedence over shape similarity. On the other hand, it is possible that the infants apply both of these principles to word-object association and perceptual categorization as the situation demands. The higher correct rate for the naming task compared to the visual task in experiment of naming and visual-pairing task is consistent with the interpretation that infants are more conceptually driven than visual driven in the judgment of categories.

The ability to identify as belonging to the same category objects which bears no or little resemblance, and to judge objects which bears a strong resemblance to each other as belonging to different categories, are formed through the stages of the development [13]. The understanding of the concept of identity plays the prominent role in perceptual categorization.

\section{Acknowledgments}

We thank Kazuko Machida and Chizuko Ono of Early Development Activity Center (EDA), Sony Foundation for Education Tokyo for helping the setup of the experiments. We thank the participating infants and mothers of EDA for their patience and good wills.

\section{References}

[1] E. Rosch and C.B. Mervis, "Family resemblances: Studies in the internal structure of categories," Cognitive Psychology, vol.7, pp.573-605, 1975.

[2] S. Harnad, "The symbol grounding problem," Physica D, vol.42, pp.335-346, 1990.

[3] L.K. Obler and K. Gjerlow, Language and the Brain, Cambridge University Press, Cambridge, UK, 1999.

[4] N.N. Soja, S. Carey, and E.S. Spelke, "Ontological categories guide young children's inductions of word meaning: Object terms and substance terms," Cognition, vol.38, pp.179-211, 1991.

[5] S. Carey and E. Bartlett, "Acquiring a single new word," Papers and Reports on Child Language Development, vol.15, pp.17-29, 1978.

[6] E.M. Markman and J.E. Hutchinson, "Children's sensitivity to constraints on word meaning: Taxonomic versus thematic relations," Cognitive Psychology, vol.16, pp.1-27, 1984.

[7] B. Landau, L.B. Smith, and S.S. Jones, "The importance of shape in early lexical learning," Cognitive Development, vol.3, pp.299-321, 
1988.

[8] P.C. Quinn and P.D. Eimas, "Perceptual cues that permit categorical differentiation of animal species by infants," Journal of Experimental Child Psychology, vol.63, pp.189-211, 1996.

[9] P.D. Eimas and P.C. Quinn, "Studies on the formation of perceptually based basic-level categories in young infants," Child Development, vol.65, pp.903-917, 1994.

[10] T. Nazzi and A. Gopnik, "Linguistic and cognitive abilities in infancy: When does language become a tool for categorization?," Cognition, vol.80, pp.B11-B20, 2001.

[11] S.A. Gelman and E.M. Markman, "Categories and induction in young children," Cognition, vol.23, pp.183-209, 1986.

[12] S.A. Gelman and E.M. Markman, "Young children's inductions from natural kinds: The role of categories and appearances," Child Development, vol.58, pp.1532-1541, 1987.

[13] R.L. Fantz, "The origin of form perception," Scientific American, vol.204, pp.66-72, 1961.

[14] R.L. Fantz, "Pattern vision in newborn infants," Science, vol.140, pp.296-297, 1963.

[15] S. Amano and T. Kondo, Nihongo-no Goitokusei (Lexi-cal properties of Japanese), SANSEIDO Co., Ltd., Tokyo, Japan, 2003.

[16] G. Lakoff, Women, Fire, and Dangerous Things: What Categories Reveal about the Mind, The University of Chicago Press, Chicago, 1987.

[17] N.N. Soja, S. Carey, and E.S. Spelke, "Discussion: Perception, ontology, and word meaning," Cognition, vol.45, pp.101-107, 1992.

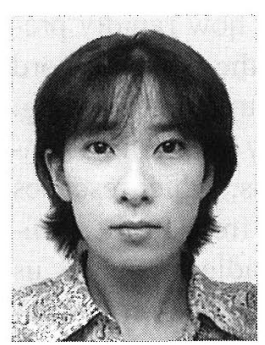

Tamami Sudo is a doctoral student at the Interdisciplinary Graduate School of Science and Engineering Tokyo Institute of Technology, Japan. She received the B.E. degree in electronic engineering from Yokohama National University in 2001 and M.S. degree in cognitive science from Tokyo Institute of Technology in 2003. Her current research interests include human communication and social cognition.

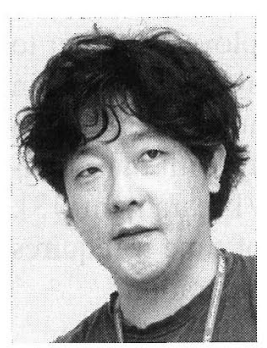

Ken Mogi is a senior researcher at Sony Computer Science Laboratories, Inc., and a visiting professor at Interdisciplinary Graduate School of Science and Engineering Tokyo Institute of Technology, Japan. He received a Ph. D in physics from University of Tokyo in 1994. After conducting research in Riken and University of Cambridge, U.K., he joined the Sony Computer Science Laboratories in 1997. His main research interest is the cognitive processes in the brain. 